Check for updates

Cite this: RSC Adv., 2017, 7, 25740

Received 31st March 2017

Accepted 7th May 2017

DOI: 10.1039/c7ra03719h

rsc.li/rsc-advances

\section{Controlled synthesis of soluble conjugated polymeric nanoparticles for fluorescence detection $\uparrow$}

\begin{abstract}
Yanan Dai, Peng Zhao, Lili Wang, Yun Ding and Aiguo Hu (D)*
Highly fluorescent soluble conjugated polymeric nanoparticles (SCPNs) were synthesized through Suzukitype polycondensation in confined nanoreactors, resulted in well-controlled particle sizes and uniform particle-size distributions. The SCPNs showed a wide range of the fluorescence emission maximum from $380 \mathrm{~nm}$ to $525 \mathrm{~nm}$. They were surface-functionalized with boronic acid groups, obtained the corresponding BA-SCPNs, and further applied to the detection of two physiologically important species glucose and $\mathrm{Fe}^{3+}$ based on their interactions with boronic acid groups. The fluorescence quenching efficiency reached up to $32.1 \%$ and $92.4 \%$ for glucose and $\mathrm{Fe}^{3+}$, respectively. Among these fluorescent probes, BA-SCPN 3 exhibited enhanced glucose sensitivity compared with fructose, which might own to the formation of $1: 1$ cyclic complexes with biboronic acid derivatives. Meanwhile, BA-SCPN 3 also showed superior $\mathrm{Fe}^{3+}$ selectivity compared with other metal ions probably due to the formation of metal hydroxides between $\mathrm{Fe}^{3+}$ and the abundant hydroxyl groups in the boronic acid.
\end{abstract}

\section{Introduction}

Fluorescent chemosensors are a class of powerful tools for both analytical sensing and optical imaging because of their high sensitivity, specificity, fast response, and technical simplicity. ${ }^{\mathbf{1 - 3}}$ Generally, a fluorescent chemosensor is composed of a fluorescent dye and a receptor, with a built-in transduction mechanism that converts recognition events into variations of the emission properties of the fluorescent component. ${ }^{4}$ Conventional fluorophores however suffer from some inherent limitations, such as photobleaching, limited brightness and so on. In the last decade, fluorescent nanoparticles like C-dots and conjugated polymer nanoparticles (CPN) have emerged as a new class of fluorophores with the potential to overcome these limitations., While most current CPNs are composed of hydrophobic linear conjugated polymers carrying no functional groups, CPN-based sensors are mostly developed by blending these conjugated polymers with fluorescent sensing materials. ${ }^{7,8}$ Therefore, fabrication of fluorescent chemosensors with tailored microstructure and high selectivity towards the species in complex mixtures still constitutes a great challenge.

Shanghai Key Laboratory of Advanced Polymeric Materials, School of Materials Science and Engineering, East China University of Science and Technology, Shanghai, 200237, China. E-mail: hagmhsn@ecust.edu.cn; Fax: +86-21-64253037; Tel: $+86-21-64253037$

$\dagger$ Electronic supplementary information (ESI) available: Details of the synthetic procedures of other SCPNs, NMR, DLS, UV-vis, and PL spectra, photostability data of BA-SCPN 3, and optical images of SCPNs. See DOI: 10.1039/c7ra03719h
Diabetes is a chronic metabolic disease, which represents major human health threats. Normally it results in some severe complications including cardiovascular disease, kidney failure, and blindness if the blood glucose lever is not controlled properly. ${ }^{9}$ Monitoring glucose concentration in blood is one of the primary challenges in the management of diabetes. At present, the popular way to detect glucose is based on glucose oxidase. But this method has several severe disadvantages, especially the low thermal stability of the enzyme during use or sterilization. ${ }^{\mathbf{1 0 - 1 2}}$ In view of these limitations, there are significant interests in searching for reliable, sensitive, inexpensive, and rapid non-enzyme sensors for the detection of glucose in blood serum or saliva. ${ }^{13}$ Besides, ferric ion $\left(\mathrm{Fe}^{3+}\right)$ is also an essential transition metal ion in most organisms and plays vital roles in biological, environmental, and chemical systems. ${ }^{\mathbf{1 4 , 1 5}}$ However, excessive $\mathrm{Fe}^{3+}$ ions in human body could lead to severe diseases such as cancers, hepatitis, hemochromatosis, and dysfunction of organs. ${ }^{16-18}$ Therefore, intense research efforts have been devoted to the development of chemosensors for $\mathrm{Fe}^{3+}$ ion detection. Thus, in this work, we aim to develop a new kind of fluorophores in order to detect both glucose and $\mathrm{Fe}^{3+}$ ion in human body.

Synthetic boronic acid derivatives have been used as receptors in the development of glucose sensing systems owning to their capability to bind covalently to 1,2- or 1,3-diols to form five- or six-membered cyclic esters. ${ }^{19-21}$ This mechanism of recognition was used by Yoon and Czarnik $^{22}$ for the development of the first fluorescent saccharide sensing system. Besides, $\mathrm{Fe}^{3+}$ can form metal hydroxides by specifically coordinating with the hydroxyl groups in boronic acid. ${ }^{\mathbf{1 4 , 2 3 , 2 4}}$ 
Therefore, it is feasible to apply the chemosensors with boronic acid groups to detect $\mathrm{Fe}^{3+}$ at the same time. Unfortunately, in most cases, the applicability of the boronic acid systems was restricted due to their limited solubility in aqueous solutions. Recently, our group reported a new method to synthesize sizecontrolled conjugated microporous polymers (CMPs) through polycondensations ${ }^{25,26}$ in confined nanoreactor, where the catalysts (palladium nanoparticles) were embedded in mesoporous supports, namely, silica-supported carbon nanomembranes (SS-CNMs). CMPs, a class of amorphous materials possessing three-dimensional networks with their building blocks linked in a $\pi$-conjugated fashion, have emerged as an important material platform since $2007 .{ }^{27}$ Later on, they are extensively utilized in gas adsorption, ${ }^{28-31}$ electrical energy storage, ${ }^{32-34}$ and especially in chemosensors ${ }^{35-37}$ owning to their high sensitivity and technical simplicity. However, the difficulty in processing and device integration have been considered as the Achilles heel for CMPs to be competitive in future applications. ${ }^{38}$ By using our catalyst@pore approach, the growth of CMPs was limited within the mesochannels of the nanoreactors, providing soluble CMPs (SCMPs) which could be easily processed for further applications. Herein, we not only employ the above mentioned method to synthesize a series of fluorescent soluble conjugated polymeric nanoparticles (SCPNs) through Suzuki polycondensation, but also extend this research through surface-modification with boronic acid groups. Therefore, the SCPNs with abundant boronic acid antennas are applied to the sensing and detection of glucose and $\mathrm{Fe}^{3+}$ thanks to their high solubility, fluorescence and stability.

\section{Experimental details}

\section{Materials and measurements}

$N, N$-Dimethylformamide (DMF) was dried over calcium hydride and distilled before use. 1,3,5-Tris(3-iodophenyl)benzene, ${ }^{39}$ tetra(4-bromophenyl)ethylene ${ }^{40}$ and 4,4'-biphenydiboronic $\operatorname{acid}^{\mathbf{4 1}}$ were synthesized according to literature procedures. The SBA-15-supported carbon nanomembrane (denoted SS-CNMs) was prepared according to our published procedure. ${ }^{42}$ Other chemicals were of commercial grade and used as received.

${ }^{1} \mathrm{H}$ NMR (400 MHz) and ${ }^{13} \mathrm{C}$ NMR (100 MHz) spectra were recorded in chloroform-d $\left(\mathrm{CDCl}_{3}\right)$ on an Ultra Shield 400 spectrometer (BRUKER BIOSPIN AG, Magnet System $400 \mathrm{MHz} / 54$ $\mathrm{mm}$ ). Fourier transform infrared analysis (FTIR) was recorded from $\mathrm{KBr}$ pallets on a Nicolet Magna 5700 FTIR spectrometer. High resolution transmission electron microscopy (HR-TEM) images were obtained on a JEOL JEM-2100 microscope (JEOL, Japan) at an acceleration voltage of $200 \mathrm{kV}$. The nanoreactor samples were prepared by drop-casting ethanol dispersion onto a copper grid. The conjugated polymer nanoparticles were dissolved in dichloromethane and drop-cast onto a copper grid. The hydrodynamic diameters of the SCPNs in DMF were analysed using a Zetasizer Nano at $25{ }^{\circ} \mathrm{C}$ with light of wavelength $633 \mathrm{~nm}$ (Malvern, UK). UV-vis absorption spectra were recorded on a Shimadzu UV-2550 UV-vis spectrometer with tetrahydrofuran (THF) as the solvent at room temperature. Fluorescence spectra were obtained on a Fluorolog-3-P UV-vis-NIR fluorescence spectrophotometer (Jobin Yvon, France). The palladium contents of the samples were analysed by ICP-AES on a Thermo Elemental IRIS 1000 instrument.

\section{Preparation of Pd@SS-CNMs nanoreactors}

$\mathrm{A}_{2} \mathrm{PdCl}_{4}$ solution in acetone $(0.5 \mathrm{M}, 1 \mathrm{~mL})$ was added into a Schlenk flask containing SS-CNMs (100 mg) under vacuum at low temperature to prevent excessive evaporation of acetone. Then the mixture was ultrasonicated for $30 \mathrm{~min}$ and evaporated at $90{ }^{\circ} \mathrm{C}$ to give a viscous solid. After freeze dried for $24 \mathrm{~h}$, the powder was gently washed with excessive deionized water. The resulting residue was reduced in hydrogen/argon (10/90) atmosphere in a tube furnace at $300{ }^{\circ} \mathrm{C}$, giving Pd@SS-CNMs with $3.4 \%$ Pd loading as checked via ICP-AES analysis.

\section{Preparation of SCPN 3}

Polycondensation. 2,2',7, $7^{\prime}$-Tetrabromo-9, $9^{\prime}$-spirobifluorene (0.095 g, $0.15 \mathrm{mmol}$ ), p-phenylenediboronic acid (0.05 g, 0.3 $\mathrm{mmol}$ ), tetrabutylammonium fluoride (TBAF, $0.426 \mathrm{~g}, 1.35$ mmol), Pd@SS-CNM (5 mg, $1.1 \mu \mathrm{mol}$ of Pd), and DMF $(2 \mathrm{~mL})$ were successively added to a $10 \mathrm{~mL}$ sealed tube under a nitrogen atmosphere. After freeze-pump-thaw for three times, the mixture was sealed and stirred at $120{ }^{\circ} \mathrm{C}$ for $120 \mathrm{~h}$.

Purification. After removal of the heterogeneous catalysts by centrifugation, the supernatant was concentrated at reduced pressure, and then added with silica gel $(4 \mathrm{~g})$. Then the silica gel adsorbed product was washed with deionized water for $12 \mathrm{~h}$ to remove TBAF, followed by Soxhlet extracted for another $24 \mathrm{~h}$ with THF. The THF extraction was concentrated and added dropwise into methanol. The precipitate formed was isolated through centrifugation and washed with methanol for three times. Pale yellow solid was obtained after drying under vacuum at $40{ }^{\circ} \mathrm{C}$ for overnight (38 mg, 60.8\%).

Other SCPNs were prepared in the same manner, detailed procedures are listed in ESI. $\dagger$

\section{The measurement of the fluorescence quantum yields}

The quantum yield $(Q)$ of the SCPN 3 was measured by comparing the integrated PL intensities and absorbance values against the reference quinine sulphate. Absorbance data of the samples and the standard was measured using a UV-visible spectrophotometer. Photoluminescence (PL) emission spectra of all the sample solutions were recorded at an excitation wavelength of $350 \mathrm{~nm}$. To minimize re-absorption effects, the absorption was kept below 0.1 at the excitation wavelength. Quantum yield was determined using the following equation:

$$
Q_{\mathrm{x}}=Q_{\mathrm{std}}\left[I_{\mathrm{x}} / A_{\mathrm{x}}\right]\left[A_{\mathrm{std}} / I_{\mathrm{std}}\right]\left[\eta_{\mathrm{x}} / \eta_{\mathrm{std}}\right]^{2}
$$

where $Q$ is the fluorescence quantum yield, subscript $\mathrm{x}$ and std refers to the sample being tested and standard reference, respectively. $I$ is the measured integrated emission intensity, $\eta$ is the refractive index and $A$ is the optical density, which is quantified via a UV-vis spectrophotometer. 


\section{Surface functionalization of SCPNs with boronic acid}

SCPN 3 (10 mg), redistilled THF ( $5 \mathrm{~mL}$ ) were successively added to a $50 \mathrm{~mL}$ Schlenk tube, which was then sealed and degassed with three freeze-pump-thaw cycles, followed by the addition of $n$-butyl lithium $(0.1 \mathrm{~mL})$, and the mixture was stirred for $2 \mathrm{~h}$ at $-78{ }^{\circ} \mathrm{C}$. After that, trimethylborate was quickly added to the mixture and further stirred for $2 \mathrm{~h}$, and finally the mixture was warmed up to $0{ }^{\circ} \mathrm{C}$ within an ice-water bath for $3 \mathrm{~h}$.

Purification. The above mixture was concentrated by evaporation, and $2 \mathrm{M} \mathrm{NaOH}(10 \mathrm{~mL})$ was added into the system at $60{ }^{\circ} \mathrm{C}$ and stirred for $1.5 \mathrm{~h}$, followed by neutralization with $2 \mathrm{M}$ $\mathrm{HCl}$. After concentrated by evaporation, THF was added and the slurry was vigorously stirred for a while and then centrifuged. The supernatant was concentrated to gain the product as a viscous solid $(8.6 \mathrm{mg}, 86 \%)$

\section{Results and discussion}

\section{Controlled synthesis of SCPNs}

The reactive palladium (0) catalyst (Pd@SS-CNMs) was prepared according to our previous procedure. ${ }^{26}$ Fig. $\mathrm{S} 1 \dagger$ shows the TEM images of the Pd@SS-CNMs. It is clear that all the palladium nanoparticles are well dispersed inside the mesochannels, no palladium nanoparticles are found on the external surface, which is essential for the controlled growth of the SCPNs.

SCPNs were synthesized between seven combinations of monomers $\left(\mathrm{A}_{x}+\mathrm{B}_{y}, x>2, y \geq 2\right)$ through Suzuki polycondensation in Pd@SS-CNMs (Scheme 1). The compounds chosen in these combinations represented the most frequently used monomers in Suzuki coupling reaction, including aryl halide and arylboronic acid. The obtained polymers are freely soluble in a variety of organic solvents, like dichloromethane,

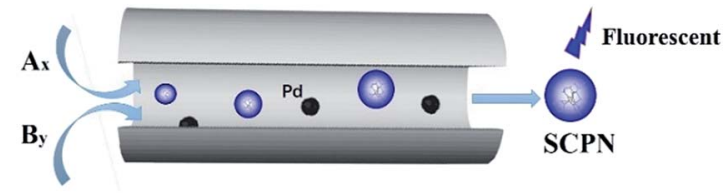

Molecular structure of monomers:
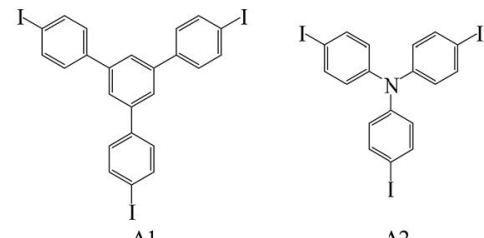

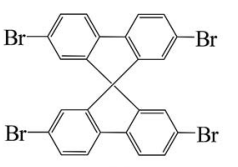

A3

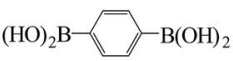

$\mathrm{B} 1$

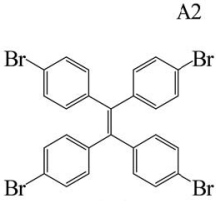

A4

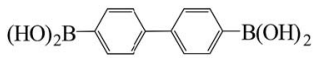

Scheme 1 Size-controlled synthesis of SCPNs in a nanoreactor and monomer combinations employed in the Suzuki polycondensation.
THF, DMF, ethyl acetate, and toluene to give transparent solutions (Fig. S2 $\dagger$ ). Importantly, all these solutions show strong fluorescence under UV irradiation of $365 \mathrm{~nm}$ (Fig. S3†).

The ${ }^{1} \mathrm{H}$ NMR spectra of all these SCPN solutions are similar to each other as there are only aromatic protons in these particles. The majority of the peaks appear between 6.7-7.8 ppm (Fig. S4 $\dagger$ ). And the ${ }^{13} \mathrm{C}$ NMR spectra also indicate their chemical structures, peaks corresponding to aromatic bonds are found between 115 and $150 \mathrm{ppm}$ (Fig. S5†), and the tetrahedral carbons are observed at $65 \mathrm{ppm}$. The structure of these SCPNs were also characterized by FT-IR spectroscopy (Fig. S6 $\dagger$ ). Absorption peaks at about 3030-3060 $\mathrm{cm}^{-1}$ (aromatic C-H stretching) and absorption bands at nearly $1600 \mathrm{~cm}^{-1}(\mathrm{C}=\mathrm{C}$ stretching bands) indicate the presence of the aromatic rings.

The particle sizes and size distributions of these SCPNs were measured with dynamic light scattering (DLS) at $25^{\circ} \mathrm{C}$ (Table 1, Fig. S7 $\dagger$ ). All the SCPNs are narrowly dispersed with particle sizes centred at approximately $4.0 \mathrm{~nm}$ for SCPN 1-SCPN 5 and $5.0 \mathrm{~nm}$ for SCPN 6-SCPN 7. The slight size difference between these two sets of SCPNs clearly reflects their structural difference. This is because that the introduction of biphenyl moiety increases the length of the repeating unit to form SCPNs with more flexible microstructure compared to those formed with phenyl units. The SCPNs are also visualized via high-resolution TEM (HR-TEM). As shown in Fig. 1 (see also Fig. S8†), all the particles are freely dispersed on the TEM support, exhibiting diameters around $4.0 \mathrm{~nm}$, consisting with the results obtained from DLS measurement. And the GPC analysis on the SCPNs reveals narrowly dispersed molecular weights (Fig. S9†). The molecular weights obtained from GPC are close to each other, in which the number average molecular weight of the SCPN 3 is about $1540 \mathrm{~g}$ $\mathrm{mol}^{-1}$, representatively. But the result might be underestimated as these nanoparticles are structurally compact than the polystyrene standards.

Table 1 The hydrodynamic diameters of these SCPNs

\begin{tabular}{llll}
\hline Entry & $\begin{array}{l}\text { Aryl halide } \\
\text { monomers }\end{array}$ & $\begin{array}{l}\text { Arylboronicchemacid } \\
\text { monomers }\end{array}$ & $\begin{array}{l}\text { DLS } \\
\text { (nm) }\end{array}$ \\
\hline SCPN 1 & A1 & B1 & 3.99 \\
SCPN 2 & A2 & B1 & 3.87 \\
SCPN 3 & A3 & B1 & 4.37 \\
SCPN 4 & A4 & B1 & 4.18 \\
SCPN 5 & A5 & B1 & 3.90 \\
SCPN 6 & A3 & B2 & 5.04 \\
SCPN 7 & A4 & B2 & 4.84 \\
& & &
\end{tabular}
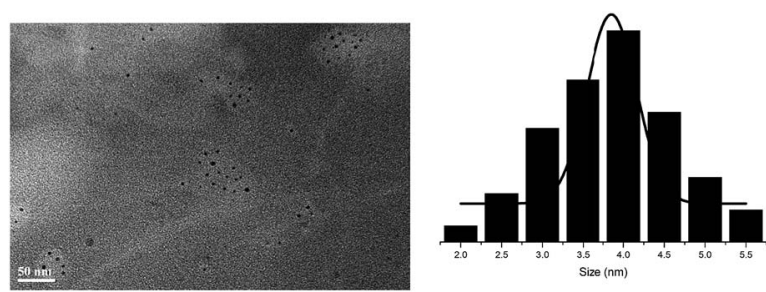

Fig. 1 TEM image (left) and size-distribution histogram (right) of SCPN3. 

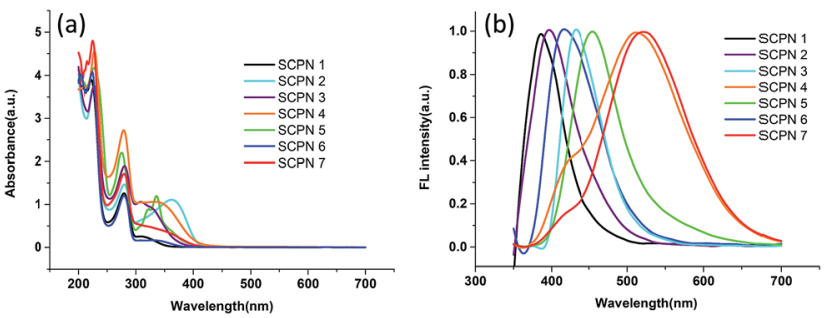

Fig. 2 (a) UV-vis spectra and (b) normalized photoluminescence spectra of SCPNs under UV irradiation at $350 \mathrm{~nm}$.

The UV-vis spectra (Fig. 2a) of all these SCPNs show a major absorbance peak appears around $275 \mathrm{~nm}$, attributing to the $\pi-$ $\pi^{*}$ transition of aromatic moieties. And the difference in the wavelength from $300 \mathrm{~nm}$ to $400 \mathrm{~nm}$ might be attributed to the difference in chemical structures of the SCPNs. The normalized photoluminescence spectra (Fig. 2b) of SCPNs show that the SCPNs have a wide range of the emission maximum from $380 \mathrm{~nm}$ to $525 \mathrm{~nm}$, owning to the difference of their fluorophore units. SCPN 3 and SCPN 4 are representative of the two typical nanoparticles at short wavelengths and long wavelengths, and the fluorescence quantum yields of them were calculated to be $19 \%$ and $11 \%$, respectively.

\section{Modification of SCPNs with boronic acid}

The SCPNs formed from the confined polycondensation are structurally similar to hyperbranched polyarylenes or can be classified as premature nano-sized gels. ${ }^{43}$ The termini (or surface) of these SCPNs are rich of functional groups (either halides or boronic acids). As demonstrated in our earlier work, most of the terminal groups are indeed halides due to the reaction mechanism of Suzuki coupling reaction. ${ }^{25}$ The aryl halides are known as a kind of reactive species possible for the introduction of other functional groups through lithiation, Grignard reagent and cross coupling with a variety of nucleophiles. In this work, we used simple lithiation strategy, followed by the addition of trimethylborate and hydrolysis to introduce boronic acid groups onto the surface of the SCPNs, obtained the corresponding BA-SCPNs. After this surface modification, the

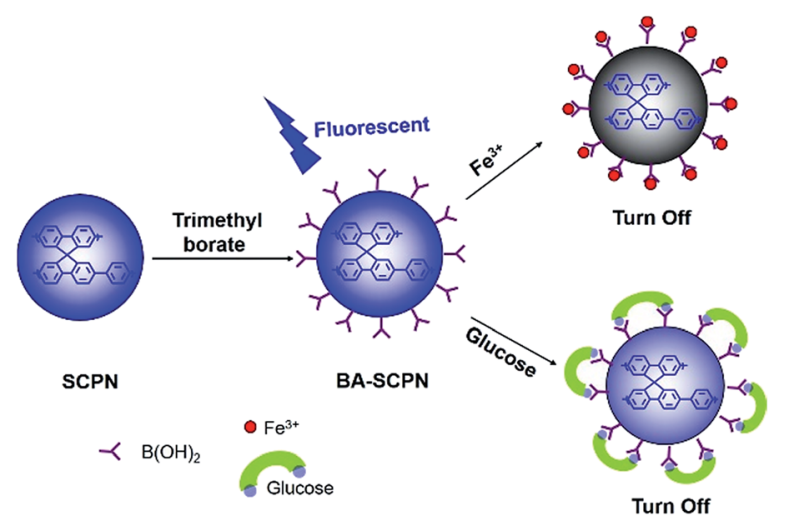

Scheme 2 Surface modification of SCPNs and the fluorescence detection with BA-SCPNs. solubility of the BA-SCPNs in aqueous solution was greatly enhanced, and can be further applied to the fluorescence sensing, including the detection of ferric ion $\left(\mathrm{Fe}^{3+}\right)$ and glucose (Scheme 2).

The UV-vis and fluorescence features of the BA-SCPNs were slightly affected (Fig. S10 $\dagger$ ) after functionalization with boronic acid. It can be seen clearly that the band broadening of both UV and FL spectra, which might be attributed to the complication of the chromophores. ${ }^{9}$ They also showed a high photostability even after $1000 \mathrm{~s}$ continuous laser scanning at $350 \mathrm{~nm}$ (Fig. S11 $\dagger$ ). The existence of the boronic acid groups in the BASCPNs was confirmed by probing the spectral changes upon reaction of the BA-SCPNs with Alizarin Red S (ARS). ${ }^{44}$ Boronic acid typically forms a complex with ARS that results in a colour change from red to yellow. The absorbance spectra of ARS alone and ARS with the addition of the BA-SCPN 3 are shown in Fig. S12. $\dagger$ The result reveals a blue shift of the absorption of the dye and clearly colour change was also observed in the presence of BA-SCPN, implying the formation of boronate-ARS complex.

\section{Fluorescence detection}

Fig. 3 shows the FL quenching of the BA-SCPN 3 with glucose of various concentrations. Boronic acid derivatives are able to bind covalently to 1,2- and 1,3-diols to form five- or six-membered cyclic esters. ${ }^{20,21}$ As a result, the boronic acid transforms from the neutral trigonal form into the anionic tetrahedral form, leading to the formation of tetrahedral boronate ester. The change in the electronic properties of boronic acid-glucose ester leads to sugar-induced spectral changes through chelation enhanced-quenching (CHEQ), ${ }^{\mathbf{9} 22,45}$ thereby quenching the fluorescence property of SCPNs. The FL intensity decreases with the increasing of the concentration of glucose, and about $32.1 \%$ FL is quenched by the addition of $80 \mathrm{mM}$ glucose for BA-SCPN 3 and $21 \%$ for BA-SCPN 4, respectively (Fig. S13 $\dagger$ ). Fig. S14 $\uparrow$ shows the Stern-Volmer plot of $F_{0} / F$ versus glucose concentration, which has a good linear relationship over a range of $0-8 \mathrm{mM}$, with a correlation coefficient of 0.991 (Fig. 3b). And the detection limit was calculated to be $13.9 \mu \mathrm{M}$ based on a $3 \delta / S(\delta$ is the standard deviation of the blank signal and $S$ is the slope of the linear calibration plot). ${ }^{\mathbf{2 4 , 4 6}}$

We further studied the FL intensity trend of BA-SCPN 3 on fructose (Fig. S15 $\dagger$ ). Interestingly, the BA-SCPN 3 shows much
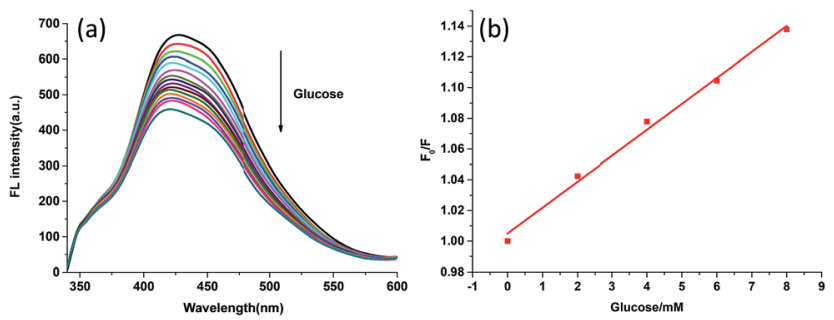

Fig. 3 (a) FL spectra of BA-SCPN 3 quenched by various amount of glucose (ranged from 0 to $80 \mathrm{mM}$ ) in a pH 9.2 PBS buffer. (b) Plot of the relative intensity of $\mathrm{FL}\left(F_{0} / F\right)$ versus the glucose concentration measured at $330 \mathrm{~nm}$. $F_{0}$ and $F$ are the FL intensity of SCPN 3 in the absence and presence of glucose, respectively. 
less fluorescence intensity sensitivity to fructose compared with glucose under the same experimental conditions. It is well documented that D-glucose tends to form $1: 1$ cyclic complexes with diboronic acids whereas $D$-fructose prefers to form $2: 1$ acyclic complexes. ${ }^{20,47-50}$ Therefore, higher sensitivity is obtained owing to the cooperative binding through the formation of 1 : 1 rigid cyclic systems between glucose and diboronic acidbased BA-SCPNs.

The response of BA-SCPNs towards $\mathrm{Fe}^{3+}$ ion was determined by titration of the BA-SCPN 3 solution with $\mathrm{Fe}^{3+}$ solution in a HEPES buffer $\left(\mathrm{pH}=7.2\right.$ ). Upon the addition of $\mathrm{Fe}^{3+}$, the fluorescence intensity of the BA-SCPN 3 was significantly quenched up to $92.4 \%$ (Fig. $4 \mathrm{a}$ ). The sharp change in the fluorescence is even visible by naked eyes when the sample was shined with a hand-held UV lamp (365 nm). From the SternVolmer plot, a good linear relationship between $\left(F / F_{0}\right)-1$ and the $\mathrm{Fe}^{3+}$ concentration is found over a range of $0-200 \mu \mathrm{M}$, with a correlation coefficient of 0.992 (Fig. $4 \mathrm{~b}$ and S16†). And the detection limit was calculated to be $0.1 \mu \mathrm{M} .^{16,24}$

As suggested by previous reports, the above phenomenon can be attributed to the fact that $\mathrm{Fe}^{3+}$ ions form metal hydroxides by specially coordinating with the abundant hydroxyl groups in boronic acid, ${ }^{\mathbf{1 4}}$ and the electrons in the excited state of BA-SCPNs transfer to the half-filled 3d orbits of $\mathrm{Fe}^{3+} .^{51,52}$ Based on the above analyses, a coordinated BA-SCPN-Fe ${ }^{3+}$ complex would be formed, which may facilitate charge transfer from SCPN to $\mathrm{Fe}^{3+}$, changing the photoinduced electron transfer (PET) process and thus lead to a significant fluorescence quenching behaviour. When $1 \mathrm{mM}$ of EDTA, a powerful metalchelator, was added into the BA-SCPN-Fe ${ }^{3+}$ mixture, the fluorescence intensity was almost completely restored (Fig. S17 $\dagger$ ). The result demonstrated that EDTA could readily abstract $\mathrm{Fe}^{3+}$ from the BA-SCPN-Fe ${ }^{3+}$ to form more stable Fe-EDTA complex, and the BA-SCPN was released. This experiment further corroborated the above coordination-induced fluorescence quenching mechanism.

Interestingly, under the same condition, the addition of many other metal ions, including $\mathrm{Fe}^{2+}, \mathrm{K}^{+}, \mathrm{Na}^{+}, \mathrm{Ca}^{2+}, \mathrm{Mg}^{2+}, \mathrm{Al}^{3+}$, $\mathrm{Ag}^{+}, \mathrm{Zn}^{2+}, \mathrm{Gd}^{3+}$ and $\mathrm{Mn}^{2+}$, only resulted in negligible fluorescence quenching. This is probably because that the abovementioned metal ions lack the empty orbits for the photoinduced electron transfer (PET) process, thus the fluorescence of
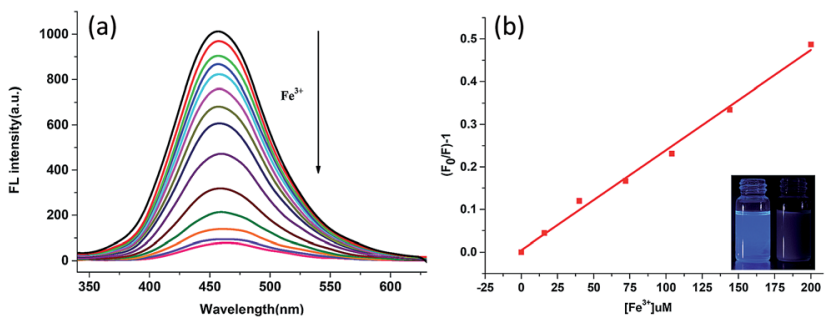

Fig. 4 (a) FL spectra of SCPN 3 quenched by various amount of $\mathrm{Fe}^{3+}$ (ranged from 0 to $800 \mu \mathrm{M}$ ) in a pH 7.2 HEPES buffer. (b) Plot of the relative intensity of $\mathrm{FL}\left(F_{0} / F\right)$ versus the $\mathrm{Fe}^{3+}$ concentration measured at $330 \mathrm{~nm} . F_{0}$ and $F$ are the FL intensity of SCPN 3 in the absence and presence of $\mathrm{Fe}^{3+}$, respectively.

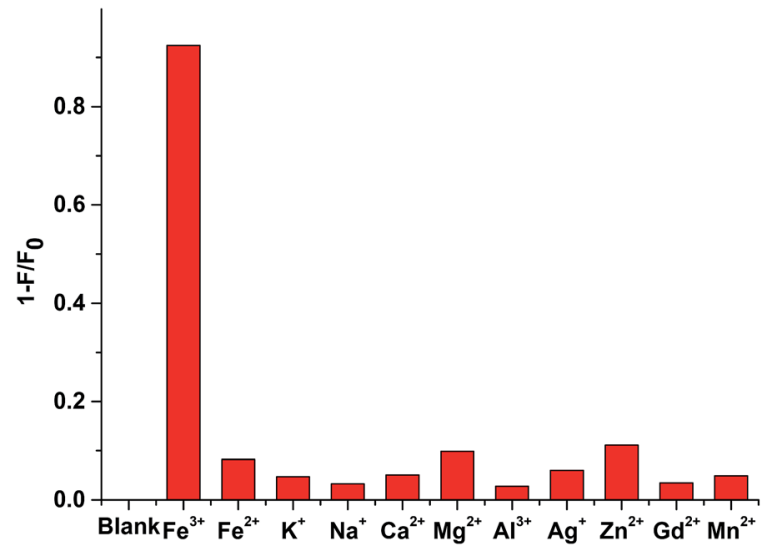

Fig. 5 Fluorescence quenching performance of the BA-SCPN 3 solution in the absence and presence of various metal ions $\left(\left[\mathrm{M}^{n+}\right]=\right.$ $800 \mu \mathrm{M}, F_{0}$ and $F$ correspond to BA-SCPN 3 fluorescence intensities at $350 \mathrm{~nm}$ in the absence and presence of ions, respectively).

the system can't be influenced. These findings validate the high sensitivity and selectivity of the fluorescent sensing of BASCPNs towards $\mathrm{Fe}^{3+}$ (Fig. 5).

\section{Conclusion}

In summary, we synthesized a series of fluorescent SCPNs through Suzuki polycondensation in a confined nanoreactor, giving a controlled particle size and size distribution. The resulting SCPNs are freely soluble in varieties of organic solvents, and show strong fluorescence under UV irradiation at $365 \mathrm{~nm}$. They were further surface-modified with boronic acid groups, and the end-functionalized boronic acid containing BASCPNs were applied to the detection of glucose and $\mathrm{Fe}^{3+}$, generating encouraging sensing results due to their high solubility, fluorescence and stability of the BA-SCPNs. The quenching efficiency reached up to $32.1 \%$ and $92.4 \%$ for glucose and $\mathrm{Fe}^{3+}$, respectively. Meanwhile, the fluorescence sensing was highly selective over other sugars or metal ions.

\section{Acknowledgements}

The authors gratefully acknowledge the financial support from National Natural Science Foundation of China (21674035) and Shanghai Leading Academic Discipline Project (B502). AH thanks the "Eastern Scholar Professorship" support from Shanghai local government.

\section{Notes and references}

1 H. Zhu, J. L. Fan, J. J. Du and X. J. Peng, Acc. Chem. Res., 2016, 49, 2115-2126.

2 R. Martinez-Manez and F. Sancenon, Chem. Rev., 2003, 103, 4419-4476.

3 T. Gunnlaugsson, M. Glynn, G. M. Tocci, P. E. Kruger and F. M. Pfeffer, Coord. Chem. Rev., 2006, 250, 3094-3117.

4 L. Bau, P. Tecilla and F. Mancin, Nanoscale, 2011, 3, 121-133. 
5 H. Goesmann and C. Feldmann, Angew. Chem., Int. Ed., 2010, 49, 1362-1395.

6 Y. Wang and A. Hu, J. Mater. Chem. C, 2014, 2, 6921-6939.

7 C. F. Wu, B. Bull, K. Christensen and J. McNeill, Angew. Chem., Int. Ed., 2009, 48, 2741-2745.

8 F. M. Ye, C. F. Wu, Y. H. Jin, Y. H. Chan, X. J. Zhang and D. T. Chiu, J. Am. Chem. Soc., 2011, 133, 8146-8149.

9 S. Kiran and R. D. K. Misra, J. Biomed. Mater. Res., Part A, 2015, 103, 2888-2897.

10 T. S. Li, K. Zhu, S. He, X. Xia, S. Q. Liu, Z. Wang and X. Y. Jiang, Analyst, 2011, 136, 2893-2896.

11 H. Wei and E. Wang, Anal. Chem., 2008, 80, 2250-2254.

12 A. Heller and B. Feldman, Chem. Rev., 2008, 108, 2482-2505.

13 H. Zhu, L. Li, W. Zhou, Z. Shao and X. Chen, J. Mater. Chem. $B, 2016,4,7333-7349$.

14 L. Xu, W. Mao, J. R. Huang, S. H. Li, K. Huang, M. Li, J. L. Xia and Q. Chen, Sens. Actuators, B, 2016, 230, 54-60.

15 Z. Q. Hu, X. M. Wang, Y. C. Feng, L. Ding, M. Li and C. S. Lin, Chem. Commun., 2011, 47, 1622-1624.

16 R. Y. Wei, Z. W. Wei, L. N. Sun, J. Z. Zhang, J. L. Liu, X. Q. Ge and L. Y. Shi, ACS Appl. Mater. Interfaces, 2016, 8, 400-410.

17 X. Huang, Mutat. Res., Fundam. Mol. Mech. Mutagen., 2003, 533, 153-171.

18 S. Toyokuni, Cancer Sci., 2009, 100, 9-16.

19 J. C. Pickup, F. Hussain, N. D. Evans, O. J. Rolinski and D. J. S. Birch, Biosens. Bioelectron., 2005, 20, 2555-2565.

20 H. Eggert, J. Frederiksen, C. Morin and J. C. Norrild, J. Org. Chem., 1999, 64, 3846-3852.

21 E. A. Moschou, B. V. Sharma, S. K. Deo and S. Daunert, J. Fluoresc., 2004, 14, 535-547.

22 J. Yoon and A. W. Czarnik, J. Am. Chem. Soc., 1992, 114, 58745875.

23 Y. Liu, Y. Liu, S. J. Park, Y. Zhang, T. Kim, S. Chae, M. Park and H. Y. Kim, J. Mater. Chem. A, 2015, 3, 17747-17754.

24 S. H. Li, Y. C. Li, J. Cao, J. Zhu, L. Z. Fan and X. H. Li, Anal. Chem., 2014, 86, 10201-10207.

25 S. Deng, J. Zhi, X. Zhang, Q. Wu, Y. Ding and A. Hu, Angew. Chem., Int. Ed., 2014, 53, 14144-14148.

26 B. Huang, P. Zhao, Y. Dai, S. Deng and A. Hu, J. Polym. Sci., Part A: Polym. Chem., 2016, 54, 2285-2290.

27 J. X. Jiang, F. Su, A. Trewin, C. D. Wood, N. L. Campbell, H. Niu, C. Dickinson, A. Y. Ganin, M. J. Rosseinsky, Y. Z. Khimyak and A. I. Cooper, Angew. Chem., Int. Ed., 2007, 46, 8574-8578.

28 R. Dawson, D. J. Adams and A. I. Cooper, Chem. Sci., 2011, 2, 1173-1177.

29 S. Ren, R. Dawson, A. Laybourn, J.-X. Jiang, Y. Khimyak, D. J. Adams and A. I. Cooper, Polym. Chem., 2012, 3, 928-934.

30 X. Liu, H. Li, Y. Zhang, B. Xu, A. Sigen, H. Xia and Y. Mu, Polym. Chem., 2013, 4, 2445-2448.
31 Y. Chen, H. Sun, R. Yang, T. Wang, C. Pei, Z. Xiang, Z. Zhu, W. Liang, A. Li and W. Deng, J. Mater. Chem. A, 2015, 3, 8791.

32 X. Feng, Y. Liang, L. Zhi, A. Thomas, D. Wu, I. Lieberwirth, U. Kolb and K. Müllen, Adv. Funct. Mater., 2009, 19, 21252129.

33 Y. Kou, Y. Xu, Z. Guo and D. Jiang, Angew. Chem., Int. Ed., 2011, 123, 8912-8916.

34 X. Zhuang, D. Gehrig, N. Forler, H. Liang, M. Wagner, M. R. Hansen, F. Laquai, F. Zhang and X. Feng, Adv. Mater., 2015, 27, 3789-3796.

35 X. Liu, Y. Xu and D. Jiang, J. Am. Chem. Soc., 2012, 134, 87388741.

36 X. Ding and B.-H. Han, Angew. Chem., Int. Ed., 2015, 54, 6536-6539.

37 M. Yu, X. Wang, X. Yang, Y. Zhao and J. X. Jiang, Polym. Chem., 2015, 6, 3217-3223.

38 F. Vilela, K. Zhang and M. Antonietti, Energy Environ. Sci., 2012, 5, 7819-7832.

39 Y. N. Zhao, J. A. Li, C. J. Li, K. Yin, D. Y. Ye and X. S. Jia, Green Chem., 2010, 12, 1370-1372.

40 B. Han, J. Wang, Q. Chen, N. Bian, X. Zhang, D. Zhou, Faming Zhuanli Shenqing, CN 102442873 A, 2012.

41 I. G. C. Coutts, H. R. Goldschmid and O. C. Musgrave, J. Chem. Soc. C, 1970, 488-493.

42 X. Yang, Z. Li, J. Zhi, J. Ma and A. Hu, Langmuir, 2010, 26, 11244-11248.

43 A. Laybourn, R. Dawson, R. Clowes, T. Hasell, A. I. Cooper, Y. Z. Khimyak and D. J. Adams, Polym. Chem., 2014, 5, 6325-6333.

44 V. Heleg-Shabtai, R. Aizen, R. Orbach, M. A. Aleman-Garcia and I. Willner, Langmuir, 2015, 31, 2237-2242.

45 P. A. Devi and M. D. Heagy, Tetrahedron Lett., 1999, 40, 78937896.

46 J. L. Pablos, S. Vallejos, S. Ibeas, A. Muñoz, F. Serna, F. C. García and J. M. García, ACS Macro Lett., 2015, 979-983.

47 V. V. Karnati, X. M. Gao, S. H. Gao, W. Q. Yang, W. J. Ni, S. Sankar and B. H. Wang, Bioorg. Med. Chem. Lett., 2002, 12, 3373-3377.

48 T. D. James, K. R. A. S. Sandanayake, R. Iguchi and S. Shinkai, J. Am. Chem. Soc., 1995, 117, 8982-8987.

49 S. Arimori, M. L. Bell, C. S. Oh, K. A. Frimat and T. D. James, J. Chem. Soc., Perkin Trans. 1, 2002, 803-808.

50 S. Arimori, G. A. Consiglio, M. D. Phillips and T. D. James, Tetrahedron Lett., 2003, 44, 4789-4792.

51 Q. S. Mei, C. L. Jiang, G. J. Guan, K. Zhang, B. H. Liu, R. Y. Liu and Z. P. Zhang, Chem. Commun., 2012, 48, 7468-7470.

52 T. V. Tam, N. B. Trung, H. R. Kim, J. S. Chung and W. M. Choi, Sens. Actuators, B, 2014, 202, 568-573. 